\title{
Cooling of Integrated Circuits Using Droplet-Based Microfluidics
}

\author{
Vamsee K. Pamula \\ Duke University \\ Box 90291, Dept of ECE \\ Durham, NC 27708 \\ +19196605294 \\ vkp@ee.duke.edu
}

\author{
Krishnendu Chakrabarty \\ Duke University \\ Box 90291, Dept of ECE \\ Durham, NC 27708 \\ +19196605244 \\ krish@ee.duke.edu
}

\begin{abstract}
Decreasing feature sizes and increasing package densities are making thermal issues extremely important in IC design. Uneven thermal maps and hot spots in ICs cause physical stress and performance degradation. Many MEMS and microfluidics-based solutions were proposed in the past. We present a cooling method based on high-speed electrowetting manipulation of discrete submicroliter droplets under voltage control with volume flow rates in excess of $10 \mathrm{~mL} / \mathrm{min}$. We also propose a flow-rate feedback control where the hot areas get increased supply of droplets without the need for external sensors and electrothermocapillary control where hot areas attract droplets due to thermocapillarity and are returned to their reservoirs by electrowetting resulting in a self-contained and a self-regulated system.
\end{abstract}

\section{Categories and Subject Descriptors}

J.2 Physical Sciences and Engineering, C.3 Special Purpose and Application-based systems - Process control systems

\section{General Terms}

Measurement, Design, Experimentation.

\section{Keywords}

Microfluidics, Droplet, Electrowetting, Hot spot, Cooling, Thermal Management, Heat Removal.

\section{INTRODUCTION}

Decreasing feature sizes and increasing package densities are making thermal issues extremely important in integrated circuit (IC) design. According to recent statistics, a large proportion of field failures can be attributed to overheating, which in turn is caused by inappropriate thermal design. Uneven thermal maps and "hot spots" in ICs cause physical stress and reduce reliability.

High die temperatures affect circuit behavior in a number of adverse ways. For example, the mean-time-to-failure due to electromigration decreases exponentially with rise in temperature.

Permission to make digital or hard copies of all or part of this work for personal or classroom use is granted without fee provided that copies are not made or distributed for profit or commercial advantage and that copies bear this notice and the full citation on the first page. To copy otherwise, or republish, to post on servers or to redistribute to lists, requires prior specific permission and/or a fee.

GLSVLSI'03, April 28-29, 2003, Washington, DC, USA.

Copyright 2003 ACM 1-58113-677-3/03/0004_..\$5.00.
High temperature also leads to performance degradation since the drive capability of MOS transistors decreases almost $4 \%$ for every $10{ }^{\circ} \mathrm{C}$ rise in temperature. Moreover, interconnect (Elmore) delay increases almost $4 \%$ for every $10{ }^{\circ} \mathrm{C}$ rise in temperature.

During the last decade, a number of techniques have been developed for low-power IC design. However, it is widely believed that the total power consumption will continue to increase, despite the use of a lower supply voltage. This increase will largely be driven by higher operating frequencies, higher overall capacitance, and larger chip size. Even at reduced operating voltages, the predicted growth rate of functionality per chip will require substantially more power than supported by known thermal packaging options. New methods are therefore needed to develop embedded cooling methods for IC-level thermal management.

\section{RELATED PRIOR WORK}

A number of techniques have been proposed in the literature for chip cooling. Initially Tuckerman et al proposed microchannel heat sinks fabricated onto the back of a silicon chip substrate [12]. Later on, other means were employed to cool the chips by: immersing the electronic chips in a pool of inert dielectric liquid [1], thermosyphons, where a liquid evaporates with applied heat and condenses dissipating that heat elsewhere, in a closed system [6], and heat pipes, where the liquid evaporates, condenses at another region and reaches the hot area through wick structures that line the heat pipes thus ensuring uniform distribution of heat [14]. These techniques are inherently unsuitable for compact, embedded systems. Thus, there remains a technology void and a pressing need for dynamically cooling hot spots in embedded ICs and compact packages used for portable applications.

The latest advances in micromachining make it an attractive technology to address the heating problem. Listed below are some of the MEMS (microelectromechanical systems) and microfluidics-based solutions for cooling the chips.

Air is used instead of liquids for cost-effective implementation and simple assembly. Some of the air-based MEMS devices include an array of microfabricated membranes actuated electromagnetically to produce air microjets for cooling of packaged ICs [2], an air impinging-jet heat exchanger [13], a micromachined microfin array heat sink to enhance hydrodynamic mixing by creating vortices to dissipate heat faster [3], and a micro capillary pumped loop where an evaporator, condenser, reservoir, and liquid lines are all integrated [8]. 
Most microfluidics-based cooling devices include micro and mini-channels to miniaturize the cooling hardware. Micro-channel cooling loops have high cooling performance, and small cooling inventory. However, large pressure gradients are required to force liquids through the micro-channels. Experiments were performed in rectangular microchannels in the laminar flow, single-phase regime with water as the cooling liquid at a flow rate of $8.26 \mathrm{cc} / \mathrm{s}$ [5]. In order to increase the heat flux from a microchannel with single phase cooling, it is necessary to increase the heat-transfer coefficient by either increasing the liquid flow rate or decreasing the hydraulic diameter. A piezoelectric cooling module based on generation of secondary droplets from a primary drop which then impinge on the hot surface. The power consumption of this device is in milliwatts [4]. Electrokinetic pumps were used to pump liquids in microchannels with flow rates less than $1 \mathrm{~mL} / \mathrm{min}$ [15].

Very high flow rates have been achieved in electrowetting transport without the use of high pressure sources. Unlike piezoelectric actuators, electrowetting based systems would have no moving parts thus preventing any reliability problems. Unlike electrokinetic actuation, electrowetting requires relatively low voltages thus reducing power consumption. The power consumption for transporting a droplet over one electrode is less than $1 \mu \mathrm{W}$.

ICs are known to have non-uniform spatial power distribution with regions of hot spots. There is not much work in the literature that addresses the problem of dynamic cooling of hot spots. A unique feature of our proposed system is the ability to cool hot spots on demand and on-the-fly.

\section{PROPOSED APPROACH}

When real estate is at premium such as in portable electronics, a self-contained, closed-loop microchannel cooling system requires a low-power, compact, reliable, and efficient pump to minimize the manufacturing costs. We propose a cooling method based on pumping nanoliters of water droplets in a single-phase forced convection.

The cooling approach is based on a novel electrowetting-based droplet transport technology. In this system, cooling liquids can be transported, in user-defined patterns, under clocked-voltage control over a 2D-array of electrodes without the need for external pumps and valves [9]. In addition, liquid flow is inherently increased with increasing temperature. Thus, local hot areas on a chip have increased cooling applied locally. In addition, a combination of thermocapillarity and electrowetting results in a self-contained (without a need for external cooling liquid supply), self-regulated (without a need for external temperature sensors or driving mechanism to locate and seek the heat source), and a minimal power consumption system (thermocapillarity consumes zero power and electrowetting consumes less than microwatts).

Each electrode set can be viewed as a pump at the nano/picoliterscale, and an array of these pumps can be used for chip cooling. A droplet on a 2D-network of such pumps will move towards hot spots by thermal propulsion and will be returned to the sink via electrowetting. This amounts to a huge network of nano/picoliter pumps to assemble droplets into architectures for cooling.

A droplet rests on a hydrophobic surface over an electrode which controls its wettability through the application of an electric field, as shown in Figure 1. The actuation mechanism is based upon surface tension gradients induced in a droplet by voltage-induced electrowetting. Using this principle, discrete droplets can be transported in a highly controlled way over an array of electrodes. High-speed propagation of a few nanoliter droplets across twodimensional arrays of planar electrodes has been demonstrated at switching rates of up to $1 \mathrm{kHz}$, which corresponds to a maximum linear velocity of about $10 \mathrm{~cm} / \mathrm{sec}$. Such an array can be reconfigured to transport the droplets or hold the droplets as virtual reaction chambers where mixing can also be performed.

Since the droplets can be transported at a very high speed, heat can be removed faster. Operations such as droplet merging,

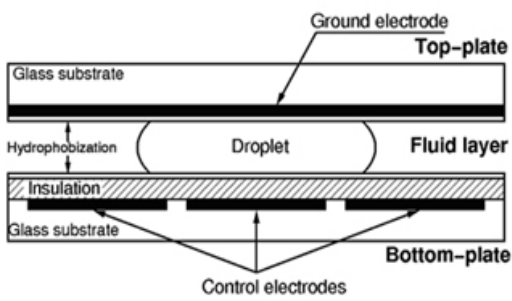

Figure 1 Cross section of an electrowetting actuator

mixing and splitting have been demonstrated [7, 10]. Aqueous droplets with a volume of $3 \mu \mathrm{L}$ over a $1.5 \times 1.5 \mathrm{~mm}^{2}$ electrode with a height of $1 \mathrm{~mm}$ can be transported at $50 \mathrm{~Hz}$. Such a droplet has a volume flow rate of $167 \mathrm{cc} / \mathrm{s}(10 \mathrm{~mL} / \mathrm{min})$ that should yield a very low thermal resistance.

The division of liquids into discrete, independently controlled packets or droplets for manipulation provides several important advantages over continuous-flow systems. We refer to this reduction of microfluidics to a set of basic repeated operations (i.e., move one unit of liquid one unit step) as "digital microfluidics". This concept allows the use of a hierarchical and cell-based design approach.

Since the only active component is embedded within a planar surface of the device, no additional components or structures such as pumps, valves, and reaction chambers are required. The absence of permanently fixed structures allows this system to be completely virtual and reconfigurable. The use of discrete droplets, in contrast to a continuous flow system, also eliminates the need for complex fabrication and assembly processes.

\section{RESEARCH DEMONSTRATIONS}

We have recently been successful in dispensing droplets from an on-chip reservoir. A liquid protrusion or a finger connected to the liquid source is first created by energizing a path of electrodes adjacent to the source. After the liquid front has extended across the energized electrodes, one or more of the intermediate electrodes is grounded, causing the finger to break at the grounded electrodes and leaving a newly formed droplet on the terminal energized electrode.

Droplet generation was also demonstrated using a continuous flow of liquid driven by external pressure pumps. A pump that delivered a continuous flow of liquid at various flow rates was coupled directly to the electrodes. This continuous flow was discretized into droplets using electrowetting. A few $10 \mathrm{~s}$ of nanoliter droplets can be formed by this method with $2 \%$ droplet volume variability. A capacitive feedback method has been 
developed that allows for dynamic control of the droplet volume by yielding the droplet footprint on the electrode based on capacitance measurements on the respective electrodes [11].

As the temperature of the electrowetting chip is increased, the threshold voltage decreases, allowing for faster velocity of the moving droplet in oil. Figure 2 shows the voltage as a function of the temperature over a few experimental runs. The voltage reported is that which is measured to oscillate a $1.7 \mu \mathrm{L}$ droplet at 5 $\mathrm{Hz}$ over 4 electrodes. The data show that the voltage required to maintain the oscillation of the droplet at $5 \mathrm{~Hz}$ reduces with increasing temperature. Conversely, at a fixed voltage and increasing temperature, the droplet moves faster. The voltage reduces by as much as $30 \%$ for a $50{ }^{\circ} \mathrm{C}$ increase in temperature.

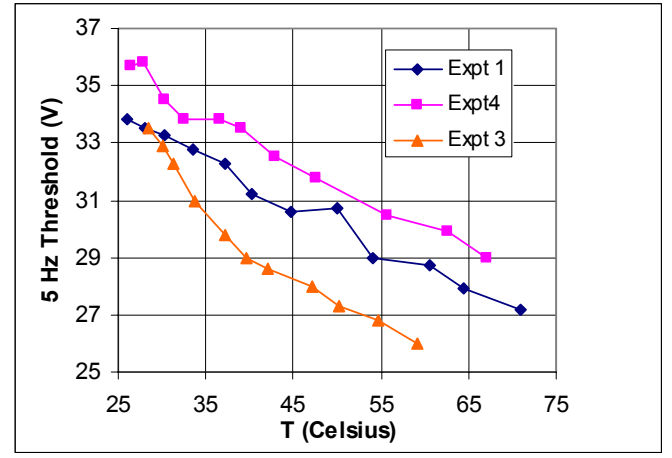

Figure 2 Actuation voltage as a function of temperature

We have recently designed and tested a digital microfluidics platform based on printed circuit board (PCB) technology as a means to rapidly prototype and inexpensively mass fabricate these devices. The platforms were fabricated by a commercial PCB manufacturer without any special modifications to the commercial process. The electrodes were made in the copper layer while the soldermask served as an insulator. The only post-processing step included spin coating the PCB chip with Teflon AF to render it hydrophobic.

\section{APPLICATION TO CHIP COOLING}

It is necessary to develop a thermal management strategy involving convective cooling by the use of droplets in contact with a thermal source. We have already demonstrated that we can dynamically vary droplet flow rates from nanoliters/minute to milliliters/minute on our digital microfluidics platform. We are investigating three mechanisms for droplet flow control. These methods offer interesting trade-offs in terms of flexibility, sensing, and computation overhead.

Thermal sensor feedback control - This method envisions an array of temperature sensors whose outputs are used to provide feedback to the droplet flow control system. Flow rates and pathways are dynamically adjusted based on the thermal profile on the chip. This "active", completely regulated approach offers the maximum flexibility (dynamically adjust flow rates based on sensor readings) but it requires overhead for sensing, computation and electronic control.

Flow-rate feedback control - In this method, the flow direction is regulated but the flow rate is controlled by temperature. Droplets are generated from a reservoir on the chip without an external supply of liquid. They flow in predefined pathways over the substrate that needs to be cooled. As the droplet flow rate due to electrowetting increases with temperature at a constant voltage, it results in faster and efficient heat removal in the presence of a thermal gradient ensuring that hotter areas get more number of droplets. The capacitive flow-rate detection system can be used to control the droplet generator based on the detected flow-rates. As a result, the overhead for sensing and computation is minimized.

Electrothermocapillary control - This method utilizes both thermocapillary-driven flow and electrowetting to achieve thermal self-regulation of droplet flows without the need for any external feedback mechanism to sense the hot spots. The droplets are so small that surface tension becomes a major force in comparison to bulk forces, and a temperature gradient induces a pressure difference across the length of the droplet. Since the droplet would be on a hydrophobic substrate, due to thermocapillary action, it will move towards the heat source (hot spot). The heated droplet can then be removed from the hot spot by electrowetting and returned to the heat sink. There is no external mechanism to drive the droplets towards the heat source; we have utilized the undesirable hot spot to attract the cooling droplets towards it and this requires zero energy to perform the work of moving the droplets. These droplets through electrowetting will be returned to a reservoir to dissipate heat, which consumes less than a few microwatts of power. This technique is the most challenging among the three mentioned here and also most desirable since power is consumed only in returning the droplets to the heat sink and placing them at strategic locations on the chip. The power consumption in this scheme would be much less than the power consumption of the previous two schemes. Droplet volumes would typically be in the nanoliter regime. Open problems to be resolved include the design of protocols for droplet routing, and integration issues at the chip/package levels.

One way of realizing this method of cooling at the package level, would be a clip-on to which any heat producing chip can be attached for heat removal. In the embodiment as a clip-on, the digital microfluidic heat removal system can be designed on the printed circuit board (PCB) over which the hot chips will be mounted, as we have already demonstrated the feasibility of using a commercially available PCB process to design the electrowetting chips.

In Figures 3 and 4 are shown the schematic top and side views respectively, of a PCB based cooling system. The system consists of reservoirs of water sandwiched between a PCB containing many arrays of linear electrowetting electrodes and a top plate. The droplets are formed out of the reservoir and transported across these arrays. The top plate consists of a thin copper foil on top of which resides the chip to be cooled. Since this copper layer will be very thin and coated with a $50 \mathrm{~nm}$ Teflon AF, it can be assumed to be fully conductive to the heat generated. Each droplet that moves across these electrodes would carry away some heat with it. Since the digital microfluidics is made on a PCB, other control electronics can also be integrated on to the same board. However, PCBs have their limitations such as very large resolutions (minimum feature size is $50 \mu \mathrm{m}$ ) and very thick solder masks and metal layers which may have potential reliability problems. 


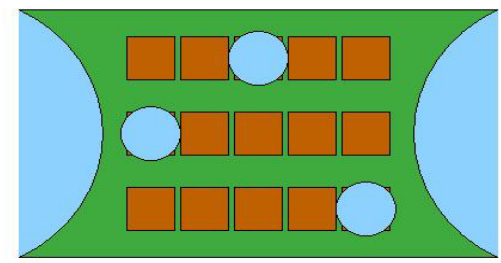

Figure 3 Top view of a PCB-based droplet cooling

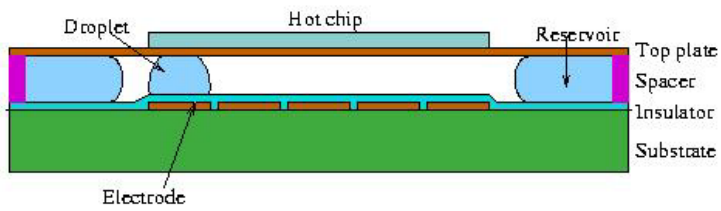

Figure 4 Cross section view of a PCB-based droplet cooling system

As an example, a die with an area of $1.5 \times 1.5 \mathrm{~cm}^{2}$ and dissipating $200 \mathrm{~W}$ would give a power density of $89 \mathrm{~W} / \mathrm{cm}^{2}$. There will be 10 electrodes in each array with a pitch of $1.5 \mathrm{~mm}$ with about 7 such arrays. The power dissipation per a single electrode can be calculated as $1.9 \mathrm{~W}$. If this power is dissipated into the droplet with a temperature rise of about $90{ }^{\circ} \mathrm{C}$, the volume flow rate required to transport this heat away by the droplets would be about $7 \mu \mathrm{L} / \mathrm{sec}$. With an electrode pitch of $1.5 \mathrm{~mm}$ and droplet height of $1 \mathrm{~mm}$, we have demonstrated volume flow rates of about $170 \mu \mathrm{L} / \mathrm{sec}$ which is almost 24 times the required flow rate. The fluid reservoirs around the PCB would provide the droplets to carry the heat away and also as heat sinks for the droplets. The direction of flow in each channel can be independently controlled according to the local needs of cooling.

\section{CONCLUSIONS}

We have presented a new chip cooling method based on highspeed electrowetting manipulation of discrete sub-microliter droplets under voltage control without any external pumps and valves. We have demonstrated electrowetting-based droplet transport on a commercially available printed circuit board (PCB) process as an inexpensive solution to fabricate the cooling system. We have also demonstrated that the liquid flow is inherently increased with increasing temperature ensuring that local hot areas on a chip have increased cooling applied locally without the need for external sensors. A combination of thermocapillarity and electrowetting is proposed as a low power solution to the heating problem.

\section{ACKNOWLEDGMENTS}

Our thanks are due to Dr. Michael Pollack, Dept. of Electrical Engineering, Duke University, for technical discussions.

\section{REFERENCES}

[1] A.E. Bergles and A. Bar-Cohen, "Direct liquid cooling of microelectronic components," In Adv. in thermal modeling of electronic components and systems, v. 2, 241-250, 1990.
[2] J.S. Campbell Jr. et al., "Thermal management of a laptop computer with synthetic air microjets," Intersociety Conf. Thermal and Thermomechanical Phenomena in Electronic Systems, 43-50, 1998.

[3] J.S. Go et al., "Heat transfer enhancement using flowinduced vibration of a microfin array," Sensors and Actuators A 90, 232-239, 2001.

[4] S.N. Heffington, et al., "Vibration-induced droplet atomization heat transfer cell for high-heat flux applications," Intersociety Conf. on Thermal and Thermomechanical Phenomena in Electronic Systems, 408412, 2002.

[5] P.S. Lee et al., "Experimental study on laminar heat transfer in microchannel heat sink," Intersociety Conference on Thermal and Thermomechanical Phenomena in Electronic Systems, 379-386, 2002.

[6] S.S. Murthy, et al., "Single chamber compact thermosyphons with micro-fabricated components," Intersociety Conf. Thermal and Thermomechanical Phenomena in Electronic Systems, pp. 321-327, 2000.

[7] P. Paik et al., "Electrowetting-based droplet mixers for microfluidic systems", Lab Chip, 3, 28-33, 2003.

[8] K. Pettigrew, et al., "Performance of a MEMS based micro capillary pumped loop for chip-level temperature control," IEEE Int. Conf, MEMS, pp. 427-430, 2001.

[9] M. Pollack et al., "Electrowetting-based actuation of liquid droplets for microfluidic applications," Applied Physics Letters, vol. 77, no. 11, 1725-1726, 2000.

[10] M.G. Pollack et al., "Electrowetting-based Actuation of Droplets for Integrated Microfluidics," Lab Chip, 2, 96-101, 2002.

[11] H. Ren et al., "Micro/nano liter droplet formation and dispensing by capacitance metering and electrowetting actuation," IEEE Conf. on Nanotechnology, 369-372, 2002.

[12] D.B. Tuckerman and R.F.W. Pease, "High-performance heat sinking for VLSI," IEEE Electron Devices Letters, vol. 2, 126-129, 1981.

[13] S.Wu et al., "Micro heat exchanger by using MEMS impinging jets," Proc. IEEE MEMS Conference, 171-176, 1999.

[14] H. Xie et al., "The use of heat pipes in personal computers," Intersociety Conf. Thermal and Thermomechanical Phenomena in Electronic Systems, pp. 442-448, 1998.

[15] S.Zeng et al., "Electroosmotic flow pumps with polymer frits," Sens. and Actuators B 82, 209-212, 2002. 